\title{
Evaluación de la calidad del servicio por medio de seis sigma en un centro de atención documental en una universidad
}

\author{
Enrique J. Delahoz-Dominguez ${ }^{(1)^{\star}}$, Tomás J. Fontalvo ${ }^{(2)}$, y Orianna M. Fontalvo(3) \\ (1) Facultad de Ingenierías, Programa Ingeniería Industrial, Universidad Tecnológica de Bolívar, Campus de Ternera, \\ Cartagena. Colombia. (correo-e: edelahoz@utb.edu.co) \\ (2) Programa de Administración Industrial, Universidad de Cartagena, Cartagena, Colombia. \\ (correo-e: tfontalvoh@unicartagena.edu.co) \\ (3) Facultad de Ingenierías, Programa de Ingeniería Industrial, Univ. del Norte, Barranquilla, Colombia. \\ (correo-e: oriannaf@uninorte.edu.co)
}

${ }^{*}$ Autor a quien debe ser dirigida la correspondencia

Recibido Jul. 29, 2019; Aceptado Sep. 24, 2019; Versión final Nov. 5, 2019, Publicado Abr. 2020

\section{Resumen}

Esta investigación propone un método de evaluación de la calidad del servicio en un centro de atención documental en una universidad. Desde un enfoque cuantitativo y racional se estableció un método fundamentado en las teorías de calidad del servicio y el concepto de Seis sigma. Se analizaron dos periodos de estudio y se contextualizaron las métricas asociadas con los criterios de evaluación. Se determinaron tres métricas de Seis Sigma, valor de defectos por partes por millón (DPMO), el nivel Z y el rendimiento. En los resultados, todas las dimensiones evaluadas en febrero presentaron un nivel de sigma mayor con respecto al que obtuvieron en enero, así como una disminución de los defectos por partes por millón de un periodo al otro, es decir mejoró la calidad del servicio y se disminuyeron costos, salvo para la dimensión de calidad 'Respuesta pertinente'. Adicionalmente, mediante el cálculo del rendimiento se encontró, que para la mayoría de los criterios de evaluación de la calidad se mantuvieron por encima del $90 \%$. Esta investigación evidencia la pertinencia de articular conceptos de calidad del servicio con las métricas de Seis Sigma, permitiendo diseñar un estándar de rendimiento del servicio para el mejoramiento de la calidad.

\section{Evaluation of service quality by means of six sigma in a center of documentary attention in a university}

\begin{abstract}
This research proposes a method to evaluate service quality in a documentary attention center in a university. From a quantitative and rational approach, a method based on the theories of quality of service and the concept of Six Sigma was established. Two study periods were analyzed and the metrics associated with the evaluation criteria were contextualized; Three metrics of Six Sigma, value of defects per parts per million (DPMO), level $Z$ and performance were determined. In the results, all the dimensions evaluated in February presented a sigma level higher than that obtained in January, as well as a decrease in the defects per parts per million from one period to the other, that is, the quality of the service improved and costs decreased, except for the quality dimension 'Relevant response'. Additionally, by calculating the yield it was found that for most of the quality evaluation criteria they remained above $90 \%$. This research demonstrates the relevance of articulating concepts of quality of service with Six Sigma metrics, allowing to design a standard of service performance for the improvement of quality.
\end{abstract}




\section{INTRODUCCIÓN}

La evolución de la forma como las empresas se relacionan con sus clientes de la mano de las nuevas tecnologías de la información, hace necesario enfatizar en la gestión del factor servicios como un aspecto estratégico para el desarrollo de las organizaciones, considerando que en un mundo de negocios tan competitivo como el actual la diferencia puede estar en una correcta interpretación de las inconformidades de los clientes y la efectividad para traducir dichas inconformidades en acciones de mejoramiento. Es así como hoy en día una de las áreas de mayor impacto en las organizaciones está asociada con la prestación de servicios. En este sentido, siempre que se evalúan y analizan métodos para el control de los servicios se genera un impacto positivo en el nivel de satisfacción y en las expectativas de quienes lo reciben. También estos métodos de control repercuten en el desempeño de las organizaciones que las prestan, por lo tanto, tener métricas asociadas al desempeño y a las dimensiones de atención de los usuarios finales surge como necesidad para mejorar la prestación de los servicios en las instituciones de educación superior.

Los centros de atención de documentación son un mecanismo para la recepción, análisis, solución y atención de los usuarios. Por esta razón en esta investigación se realiza un análisis de como una universidad mide las dimensiones de calidad del servicio, por medio de métricas que evalúan el desempeño y la evolución de la institución educativa. Muchos estudios han tratado de analizar el desempeño de los servicios prestados por medio de diferentes técnicas, en la investigación de los autores (Hongoro et al., 2005) se muestra cómo el uso de un enfoque prospectivo para medir la calidad de los servicios para un diagnóstico específico puede generar información útil para mejorar la calidad de los servicios en entornos con tecnología e información limitada. Sin embargo, llegar a medir la calidad de un servicio prestado requiere rigurosidad de cómo se lleva un criterio subjetivo a uno racional y cuantificable, en la calidad del servicio no puede olvidarse que las expectativas de los usuarios son dinámicas (Otálora et al., 2011), así mismo, es relevante mencionar que la medición de la calidad de un servicio requiere de un análisis detallado de los criterios a evaluar dependiendo del sector; en estudios realizados a contextos similares (Torres, 2010) se comprobó que la calidad del servicio es una variable latente y no solo multiatributo; es decir, es una variable compuesta por diferentes dimensiones interrelacionadas.

Considerando todo lo anterior y teniendo en cuenta que el objeto de estudio es el centro de atención documental, el cual está definido como una unidad de prestación de servicio y atención a los usuarios, que recepcióna las solicitudes documentales asociadas con la prestación del servicio universitario, para lo cual ha definido un proceso de prestación, al cual en los últimos periodos se le ha venido verificando 5 dimensiones de calidad asociados con el proceso de prestación del servicio. Y considerando que se requiere evaluar la calidad del servicio de este, surgen los siguientes interrogantes a resolver como resultado de esta investigación: ¿Cómo evaluar la calidad del servicio en un centro de atención documental, en una universidad? ¿Como identificar las dimensiones de calidad de evaluación del servicio del centro de atención documental? ¿Cómo contextualizar las dimensiones de calidad del servicio documental a las métricas de Seis Sigma con respecto a los parámetros del estudio? ¿Cuál es el rendimiento $\mathrm{Y}$ y nivel sigma $Z$ del servicio prestado por el centro de atención documental de la institución educativa por periodo? Teniendo en cuenta lo planteado anteriormente, se define el propósito de esta investigación el cual se asocia a establecer un método que permita evaluar, medir y analizar las dimensiones calidad del servicio prestado en un centro de atención documental en una organización educativa basándose en la herramienta de Seis Sigma y sus métricas. Considerando las preguntas problemas se establecieron los siguientes objetivos específicos: i) Identificar las dimensiones de calidad del proceso de prestación del servicio en un centro documental ii) Contextualizar las métricas de seis sigma de rendimiento y nivel sigma a las dimensiones de calidad iii) Evaluar las métricas de Seis Sigma relacionadas a las dimensiones de calidad del proceso de prestación del servicio iv) Comparar los resultados de evaluación de $\mathrm{Y}$ y $Z$ obtenidos en los dos periodos para analizar el desempeño del centro de atención documental.

\section{OTROS ANTECEDENTES}

En el marco de este trabajo, hay tres conceptos que requieren ser presentados y analizados: calidad en el servicio educativo, seis sigma en el servicio, y seis sigma en la educación superior

\section{Calidad en el servicio educativo}

La calidad en relación con la gestión en educación fue trasladada del ámbito empresarial y fue vinculada a la idea de control de procesos (Tumino y Poitevin, 2014). Sin embargo, el ámbito educativo es cada vez más reconocido como una industria de servicios, y esto pone un mayor énfasis en satisfacer las expectativas y necesidades de los clientes participantes (Boon, 2016). En otras investigaciones los autores (Yildiz y Kara, 2015) señalan que, aunque las entidades educativas no se enfrentan a condiciones de mercado como empresas comerciales, explican que aquellas instituciones que no brindan servicios de alta calidad serán 
marginadas y sus esfuerzos efectivos para alcanzar los objetivos de la organización se pondrán en peligro. Las instituciones educativas hoy en día han aumentado su interés por mejorar la calidad de los servicios que prestan debido a la necesidad de un constante mejoramiento que ha traído consigo la sociedad, estos servicios pueden ser de atención, solución, recepción de información, entrega de documentos, entre otros. Es también de gran importancia, generar métricas que permitan establecer valores deseables para la calidad del servicio, como se concluyó en el estudio del autor (Yousapronpaiboon, 2014), en donde este señala el análisis de la brecha entre las percepciones y las expectativas del servicio. Lo que mostró que todas las puntuaciones de las percepciones eran más bajas que las de las expectativas, lo que indica que hay muchos esfuerzos por mejorar el servicio que deben cumplirse para mejorar la calidad integral de todas sus dimensiones.

En un estudio realizado por (Rodríguez-Ponce et al, 2011) se vinculó la calidad del servicio relacionado con el concepto de sistemas de aseguramiento de la calidad de centros educativos donde se determinó que la relación es inversa, de manera que al aumentar los años de acreditación se disminuye el índice de defectos. Similarmente, otros autores (Malik et al., 2010) han explorado el impacto de la calidad del servicio en la satisfacción de los estudiantes en Institutos de educación superior de Punjab, encontrando que la calidad del servicio influye en la satisfacción de los estudiantes en múltiples dimensiones. En esta línea de ideas, las metodologías empleadas para evaluar y aproximarse a el nivel de calidad de los servicios educativos han sido muy diversas, en una investigación previa (Gadea-Cavazos, 2018) se empleó el modelo SERVQUAL al ámbito educativo, para evaluar la calidad del servicio educativo en una entidad de México. Similarmente, se han aplicado métodos combinados para medir la calidad del servicio, como en la investigación de (Abdullah, 2006) donde se comparan las metodologías HEdPERF y SERVPERF en un entorno de educación superior para determinar cuál instrumento tenía mejor unidimensionalidad, fiabilidad, validez y la varianza explicada. Además, se ha concluido por diferentes autores (Rodrigues, 2011) que la implementación de técnicas que permitan evaluar el rendimiento de los servicios facilita la identificación de las fortalezas y debilidades de su aplicación y de esta manera brindar a los proveedores de servicios la implementación de una gestión de calidad de manera más efectiva.

\section{Seis Sigma en el servicio}

La satisfacción del cliente y la competitividad están en el centro de atención para cualquier práctica de mejora de la calidad y medición del rendimiento (Shokri, 2013). La aplicación de la metodología de Seis Sigma ha permitido mejorar la evaluación de distintos sectores asociada a eficiencia, calidad, rendimiento, entre otros criterios que surgen como prerrequisito al momento de analizar cualquier contexto organizacional. Seis Sigma es una metodología potente que, en última instancia, ayuda a reducir los costos debido a la prevención de defectos y la mejora de los productos y procesos, que conducen a un aumento de la rentabilidad. (Surange, 2015). Lean Seis Sigma tiene como objetivo el estudio del "flujo de valor", que utiliza herramientas estadísticas para analizar la estabilidad del proceso tecnológico para reducir el desperdicio y la variación del proceso (Chen, 2013). Así mismo, la relación entre Seis Sigma y el servicio en algunas investigaciones (Hsieh et al., 2012) se nombra como Servicio transaccional de Seis Sigma debido a que brinda a las organizaciones un enfoque disciplinado para mejorar la eficiencia del servicio (es decir, ahorrar tiempo y costos) y su eficacia.

Teniendo en cuenta la naturaleza de este método, el acercamiento de esta herramienta al servicio ha generado resultados favorables, como se puede evidenciar en (Bo et al., 2013) donde se prueba que Seis Sigma se puede aplicar en la industria de servicios. Y proponen algunas sugerencias para que la implementación en la industria de servicios sea más efectiva. La mayoría de las investigaciones previas sobre Lean Seis Sigma se centraron en diversas perspectivas de Seis Sigma, la filosofía Lean y luego el papel de Lean Seis Sigma en la sostenibilidad, pero nunca prestaron mucha atención en el campo del sector de servicios (Deshmukh et al., 2019). En la investigación de (Rodríguez, 2015) el uso de esta herramienta en una empresa de servicios permitió establecer cuáles son las principales fallas que presenta el diseño y desarrollo del servicio que orienta la satisfacción de los clientes. También se puede encontrar en el estudio de (Chen et al., 2010) como se aplica Seis Sigma para mejorar la calidad del servicio y aumentar la competitividad de la industria de servicios. En un contexto diferente el autor (Miniati, 2015), aplicó Seis Sigma en los servicios, especialmente en relación con el desempeño de la oficina y la percepción de la calidad de los usuarios. Todo lo planteado anteriormente, da sentido a este trabajo de investigación que aborda un análisis de un centro de servicio documental y se contrastan los conceptos de calidad del servicio y Seis Sigma para una institución de educación superior.

\section{Seis Sigma en la educación superior}

La metodología Seis Sigma aún no ha sido ampliamente adoptada por las instituciones universitarias, en general para (Sporn, 2001) las empresas son lentas para adaptar nuevas formas de gestión en sus procesos. Sin embargo diferentes estudios se han desarrollado al respecto, por ejemplo (Anthony y Antony, 2017) en su investigación comparan 21 universidades del Reino Unido con 17 universidades del resto del mundo, 
encontrando diferencias en cuanto al alcance de la implementación de los procesos Seis Sigma, considerando Seis Sigma solo para procesos administrativos y no para mejorar aspectos de la docencia y la investigación. Por su parte, ( Jiju, 2017) identifica como barreras para la implementación del Seis Sigma en las Universidades la ausencia de datos de calidad, miedo a la estadística, barreras culturales y el concepto erróneo del Seis Sigma como una herramienta exclusiva para entornos industriales y de manufactura. Desde el punto de vista aplicado, (Subbarayalu y Al Kuwaiti, 2017) implementan un sistema de puntuación para determinar las métricas Seis Sigma en una facultad, involucrando aspectos como la opinión de profesores y estudiantes y la percepción del servicio educativo, identificando aspectos a mejorar en los procesos de enseñanza. Un aspecto importante de resaltar en la implementación de la metodología, en ámbitos universitarios es el proceso de medición, el cual no es desarrollado de manera estratégica para posteriores procesos de mejoramiento y tampoco es considerado como un aspecto crítico de la gestión universitaria (Nadeau, 2017).

\section{METODOLOGÍA}

Esta investigación fue de carácter evaluativa, para el desarrollo de esta se partió de un análisis racional que permitió definir y consolidar los usuarios participantes del estudio del centro de atención documental de la institución de educación superior. De igual manera, se analizó el proceso de atención documental de los usuarios para el cual se identificaron las dimensiones de calidad y las métricas de seis sigma para la evaluación asociadas. A partir de los parámetros a evaluar se realizó un análisis individual del servicio prestado al usuario, lo que llevó a poder tener una perspectiva global del servicio y sus dimensiones de calidad. Como fuente primaria de información se partió de los datos suministrados por el centro de atención documental de la institución de todos los usuarios en los dos periodos objeto de estudio. La población utilizada correspondió a todos los usuarios que solicitaron un servicio al centro de atención documental en dos periodos de recepción documental en el centro de atención de la universidad. Se seleccionaron estos periodos por ser los periodos del año que más solicitudes complejas y de fondo realizaron los usuarios. Lo anterior Con el fin de poder realizar una evaluación pertinente e integral que permitió comparar desempeños de calidad en dos periodos significativos. Y de esta forma poder analizar la evolución de la calidad del servicio en los periodos objeto de esta investigación. En la Figura 1 se muestra el esquema de las dimensiones de calidad a evaluar del centro de atención documental de la institución de educación superior.

Para el desarrollo de la investigación se seleccionó el proceso de prestación de servicio del centro de atención documental de la universidad, al que se le identificaron las cinco dimensiones de calidad del proceso de prestación del servicio ver Tabla 1 y Figura 1, que fueron evaluadas por medio de las métricas de seis sigmas: a) rendimiento $(Y)$ donde se espera que $Y$ sea mayor o igual a $95 \%$ y b) nivel Sigma (Z). El cual se espera que alcance valores iguales o superiores a 3. Esto con el fin de poder aseverar que se alcanzó un buen desempeño en las dimensiones evaluadas.

Tabla 1 Descripción de las actividades del proceso de prestación del servicio del centro de atención

\begin{tabular}{|l|l|}
\hline Dimensión del proceso evaluado & Descripción de la dimensión a evaluar \\
\hline Documentación completa & $\begin{array}{l}\text { Se controla que la solicitud que se recibe cumpla con las exigencias } \\
\text { del centro de atención documental }\end{array}$ \\
\hline Dirige la atención a la oficina pertinente & $\begin{array}{l}\text { De acuerdo con el tipo de solicitud, se asigna a la persona } \\
\text { competente para atender y responder la solicitud }\end{array}$ \\
\hline Respuesta pertinente & $\begin{array}{l}\text { Se verifica que la respuesta a la solicitud respondió a las } \\
\text { necesidades del usuario }\end{array}$ \\
\hline Estructura del comunicado & $\begin{array}{l}\text { Se verifica que la respuesta cumple con los protocolos establecidos } \\
\text { por el centro de atención }\end{array}$ \\
\hline Proceso & $\begin{array}{l}\text { Se contrasta que la estandarización de todas las actividades es } \\
\text { coherente con la ejecución }\end{array}$ \\
\hline
\end{tabular}

El estudio también se abordó desde una perspectiva cuantitativa apoyado en la técnica de Seis Sigma, para lo cual fue necesario recoger información cuantitativa de los servicios conformes y no conformes asociados a las solicitudes presentadas por los usuarios. Se contextualizaron las métricas asociadas al método a emplear con los criterios de evaluación del servicio planteados en la Figura 1. Se tuvo en cuenta las métricas de Seis Sigma, con lo que fue posible determinar los cálculos correspondientes, de manera que se obtuvo el valor de defectos por partes por millón (DPMO), el nivel $Z$ y el rendimiento $Y$ de todas las dimensiones del servicio del centro de atención documental. Después de emplear el método evaluativo de la calidad, y con las métricas calculadas, fue posible comparar los resultados para los periodos en cuestión, para finalmente, generar conclusiones sobre el desempeño de la entidad, así como las recomendaciones propuestas. En la Figura 2 se representa el método de evaluación de la calidad del servicio, propuesta en esta investigación. 


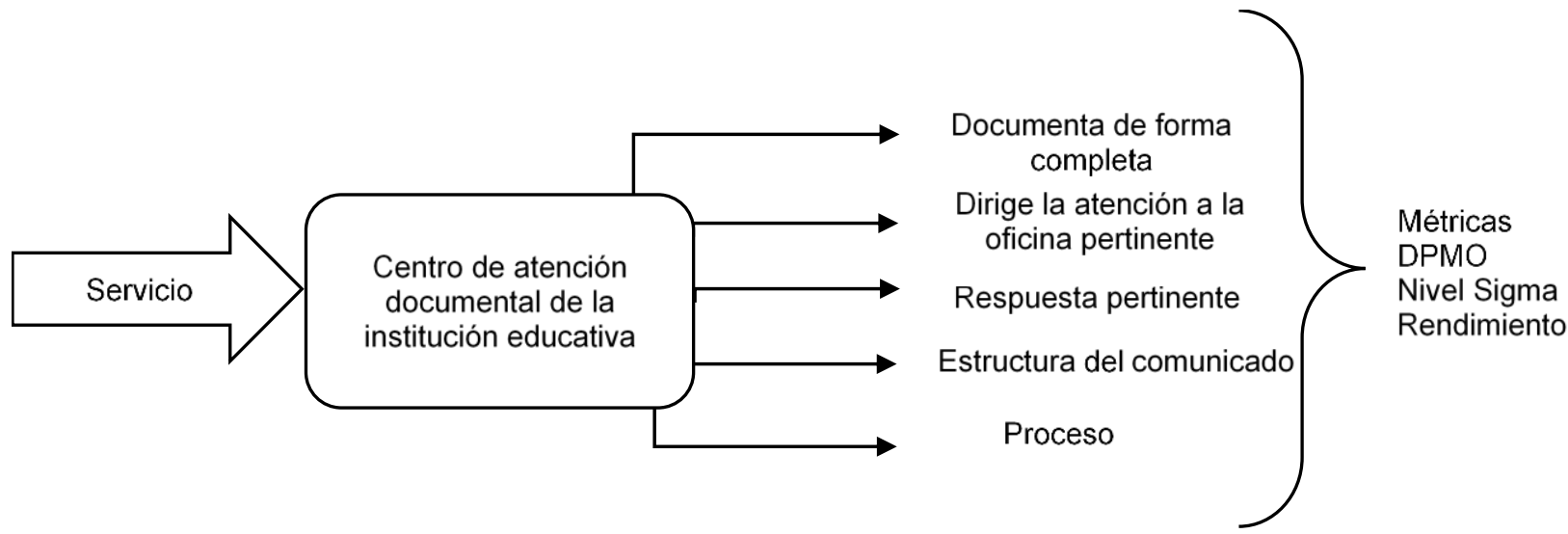

Fig. 1: Dimensiones de calidad a evaluar del centro de atención documental de la institución de educación superior

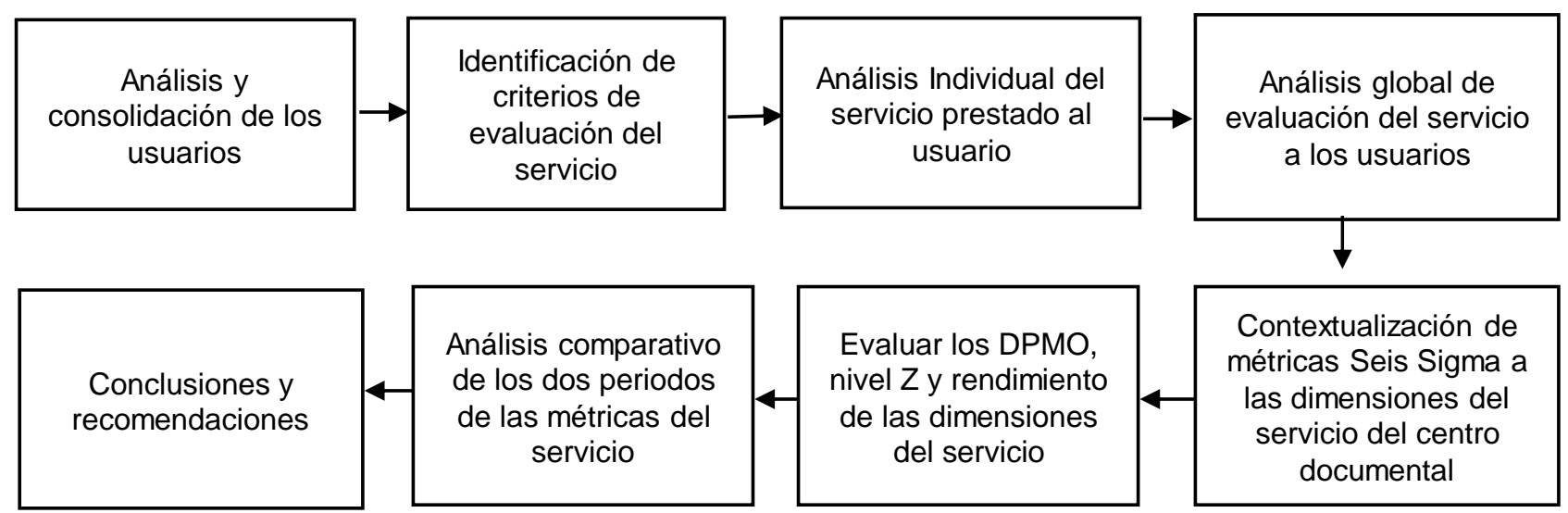

Fig. 2: Método de evaluación y análisis de la calidad del servicio

\section{RESULTADOS Y DISCUSIÓN}

Los resultados consolidados del análisis y tabulación de la prestación de servicio para las 5 dimensiones de calidad del proceso de prestación del servicio del centro de atención documental para los meses de enero y febrero de 2018 se presentan en la Tabla 2. En la Tabla 2 se observa el número de servicios solicitados para cada una de las dimensiones de calidad del servicio evaluadas, de manera que se puede realizar un análisis general sobre la relación de servicios conformes y no conformes en cada caso.

Tabla 2: Consolidado de los criterios de calidad en el servicio documental en el centro educativo

\begin{tabular}{|l|l|c|c|}
\hline \multicolumn{1}{|c|}{ Criterios } & Periodos & $\begin{array}{c}\text { Servicios } \\
\text { conformes }\end{array}$ & $\begin{array}{c}\text { Servicios No } \\
\text { conformes }\end{array}$ \\
\hline \multirow{2}{*}{ Documenta de forma completa } & Enero & 362 & 38 \\
\cline { 2 - 4 } & Febrero & 690 & 58 \\
\hline \multirow{2}{*}{$\begin{array}{l}\text { Dirige la atención a la oficina } \\
\text { pertinente }\end{array}$} & Enero & 58 & 9 \\
\cline { 2 - 4 } & Febrero & 151 & 10 \\
\hline \multirow{2}{*}{ Respuesta pertinente } & Enero & 95 & 10 \\
\cline { 2 - 4 } & Febrero & 172 & 22 \\
\hline \multirow{2}{*}{ Estructura del comunicado } & Enero & 155 & 20 \\
\cline { 2 - 4 } & Febrero & 452 & 33 \\
\hline \multirow{2}{*}{ Proceso } & Enero & 118 & 56 \\
\cline { 2 - 4 } & Febrero & 495 & 44 \\
\hline
\end{tabular}


Para el criterio 'Documenta de forma completa la gestión' se puede interpretar que los servicios inconformes para los dos periodos fueron menores en relación con la totalidad de servicios prestados. Por otro lado, para el mes de enero en la categoría de 'Proceso' se puede observar que la relación entre servicios no conformes y la totalidad requiere mejoramiento. La estructura de tabulación de los datos hace que el procedimiento de medición pueda ser replicado a otros procesos del ámbito universitario, incluso utilizando más periodos de evaluación.

\section{Métricas en la calidad del servicio documental}

Con base en la información de la Tabla 2, se establecieron las métricas de evaluación del servicio de atención documental del centro educativo. En primera instancia, para la aplicación de la metodología Seis Sigma, se definen las siguientes variables: U: Cantidad de servicios prestados; O: Oportunidad de error; n: número de servicio documental no conforme; Y: Rendimiento de la dimensión del servicio evaluada; y DPMO: Defectos de partes por millón de la dimensión del servicio documental (medida estandarizada de Seis Sigma). Las fórmulas para valorar las métricas, como son defectos en partes por millón de oportunidades (DPMO), el rendimiento (Y) y el nivel Sigma (Z), se presentan a continuación

$$
\begin{aligned}
& D P M O=\frac{n}{t} \times 1.000 .000=\frac{n}{U x O} \times 1.000 .000 \\
& Y=\left(1-\frac{n}{U \times O}\right)
\end{aligned}
$$

El cálculo para las métricas Seis Sigma del centro de atención documental de la institución de educación se consolidan en la Tabla 3. La Tabla 3 presenta las métricas de evaluación y análisis de las dimensiones de calidad del servicio documental de la entidad educativa para dos periodos. Se pude observar que la mayoría de los criterios alcanzaron el nivel mínimo Sigma $Z=3$; encontrado que el mes de febrero para el criterio 'Documenta de forma completa' obtuvo el nivel más alto $Z$ con 3.72; sin embargo, aunque en general se alcanzó el nivel esperado de $Z=3$,es decir el mínimo aceptado, la diferencia es mínima con respecto a este por lo tanto el desempeño es bueno; exceptuando el mes de enero para el criterio de 'Proceso' el cual estuvo un nivel sigma por debajo con 2.74 .

Como hallazgo de esta investigación también se observa que para todas las dimensiones evaluadas de la calidad del servicio del centro de atención documental el nivel de sigma mejoro de enero a febrero, es decir aumento, salvo para la dimensión de calidad respuesta pertinente. Lo que evidencia la gestión al interior de la institución de educación superior para mejorar el servicio del centro de atención documental y prestar un buen servicio a los usuarios de la institución.

\begin{tabular}{|c|c|c|c|c|c|c|}
\hline Criterios & Periodo & Parámetros & DPMO & Z (Sigma) & Rendimiento $Y$ & $\begin{array}{l}\text { Rendimiento } \\
\text { promedio }\end{array}$ \\
\hline \multirow[t]{2}{*}{$\begin{array}{l}\text { Documentació } \\
\mathrm{n} \text { de forma }\end{array}$} & Enero & $\begin{array}{l}n=38 ; O=6 ; \\
U=400\end{array}$ & 15833.33 & 3.65 & $98.41 \%$ & \multirow[t]{2}{*}{$98.55 \%$} \\
\hline & Febrero & $\begin{array}{l}\mathrm{n}=58 ; \mathrm{O}=6 ; \\
\mathrm{U}=748\end{array}$ & 12923.35 & 3.72 & $98.70 \%$ & \\
\hline \multirow{2}{*}{$\begin{array}{l}\text { Dirige la } \\
\text { atención a la } \\
\text { oficina } \\
\text { pertinente }\end{array}$} & Enero & $\begin{array}{l}\mathrm{n}=9 ; \mathrm{O}=5 ; \\
\mathrm{U}=67\end{array}$ & 26865.67 & 3.43 & $97.31 \%$ & \multirow[t]{2}{*}{$97.99 \%$} \\
\hline & Febrero & $\begin{array}{l}\mathrm{n}=10 ; \mathrm{O}=5 ; \\
\mathrm{U}=161\end{array}$ & 13245.03 & 3.71 & $98.67 \%$ & \\
\hline \multirow[t]{2}{*}{$\begin{array}{l}\text { Respuesta } \\
\text { pertinente }\end{array}$} & Enero & $\begin{array}{l}n=10 ; \quad O=3 ; \\
U=105\end{array}$ & 31746.03 & 3.36 & $96.82 \%$ & \multirow[t]{2}{*}{$96.51 \%$} \\
\hline & Febrero & $\begin{array}{l}\mathrm{n}=22 ; \quad \mathrm{O}=3 ; \\
\mathrm{U}=194\end{array}$ & 37800.68 & 3.28 & $96.21 \%$ & \\
\hline \multirow[t]{2}{*}{$\begin{array}{l}\text { Estructura } \\
\text { Comunicado }\end{array}$} & Enero & $\begin{array}{l}\mathrm{n}=20 ; \quad \mathrm{O}=3 ; \\
\mathrm{U}=175\end{array}$ & 38095.23 & 3.27 & $96.19 \%$ & \multirow[t]{2}{*}{$96.96 \%$} \\
\hline & Febrero & $\begin{array}{l}\mathrm{n}=33 ; \quad \mathrm{O}=3 ; \\
\mathrm{U}=485\end{array}$ & 22680.41 & 3.50 & $97.73 \%$ & \\
\hline \multirow[t]{2}{*}{ Proceso } & Enero & $\begin{array}{l}n=56 ; \quad O=3 ; \\
U=174\end{array}$ & 107279.69 & 2.74 & $89.27 \%$ & \multirow[t]{2}{*}{$93.27 \%$} \\
\hline & Febrero & $\begin{array}{l}\mathrm{n}=44 ; \quad \mathrm{O}=3 ; \\
\mathrm{U}=539\end{array}$ & 27210.88 & 3.42 & $97.27 \%$ & \\
\hline
\end{tabular}

Tabla 3. Cálculo de las métricas de Seis Sigma a las dimensiones de calidad 
De todo lo anterior, se puede aseverar que los resultados muestran que para los dos periodos el desempeño para el centro de atención documental estuvo por encima de lo esperado para un buen servicio, toda vez que las métricas calculadas para el rendimiento $Y$ y para el nivel Sigma estuvieron por encima del $95 \%$ y 3 respectivamente, lo cual evidencia que el proceso de prestación de servicio es bueno, cuando se mira de forma integral. Solo para el caso de la estandarización del proceso en enero se alcanzó un rendimiento por debajo del $95 \%$. Por lo que fue necesario acciones de seguimiento, auditoría y mejora para incrementar su buen desempeño al $97,27 \%$ en el segundo periodo.

Lo anterior es coherente con el cálculo del rendimiento, el cual para la mayoría de los criterios se mantuvo por encima del 95\%. La dimensión de calidad evaluada 'Documenta de forma completa' fue la que presentó el mayor rendimiento promedio para los dos periodos con un nivel de $98.555 \%$ y la dimensión evaluada 'proceso' fue la que obtuvo un rendimiento menor de $93.27 \%$. Adicionalmente, se puede observar según la evaluación del rendimiento, en el mes de febrero que es mayor con respecto a el de enero, lo cual muestra una mejora en términos de atención a los usuarios del centro de atención documental de la institución de educación superior. Lo que a la postre se traducen en disminución de costos, pues los defectos en partes por millón se reducen de enero a febrero. Que es la esencia que se busca cuando se utilizan este tipo de métodos de evaluación de la calidad del servicio en una organización, además de generar una estructura organizada de medición, evaluación control y mejoramiento extrapolable a otros procesos del ámbito universitario.

La relevancia de los resultados obtenidos en esta investigación se evidencian y están en concordancia con otras investigaciones similares donde también se enfocaron en la evaluación de la calidad del servicio, como se encuentra en un estudio realizado por los autores (Purnama et al., 2018) donde el resultado de la investigación muestra la disminución de defectos cuando se revisa las métricas DPMO y el nivel Sigma, de manera que el número de DPMO se redujo de 20003.75 a 11185.73, mientras que el nivel Seis Sigma demostró la mejora de 3.61 a 3.86, es decir, que la calidad del servicio de dicho proceso mejoró. Así mismo, en otra investigación realizada por el autor (Rodríguez, 2015) se encontró que la evaluación de la calidad del servicio en un contexto similar el nivel Sigma resultante fue de 1.78 y concluyen que el proceso es incapaz, lo que llevó a un punto para analizar y llevar a cabo todo un desarrollo de mejora y control. En otro tipo de contexto, la investigación de otros autores (Johnstone et al, 2019), realizado a una entidad de servicios se implementaron las métricas de defectos en partes por millón, y el nivel sigma en la calidad del servicio, se concluyó que en sistemas complejos con alto riesgo de error y poca automatización, los niveles de DPMO se encontraron entre 420,000 y 21.5 los cuales corresponden a los valores de Sigma de 1.7 a 6.

En otros contextos similares realizado por el autor (Fontalvo, 2011) en la evaluación de la calidad del servicio interno en una organización industrial, para el primer periodo analizado el nivel sigma fue de 2.5 sigma, al compararlo con el nivel sigma del periodo siguiente este mejoró a un nivel sigma de 3.1 lo cual es coherente con los resultados de esta investigación. Así mismo, en la investigación llevada a cabo por los autores (Shamsuzzaman et al, 2018) se encontró como resultado de la implementación de la metodología Seis Sigma en la calidad del servicio para una empresa de telecomunicaciones, que la reducción en la dimensión evaluativa de 'tiempo de entrega' resultó en un aumento en el nivel sigma de 0.73 a 2.66, lo que condujo a un beneficio financiero en ahorros, en disminución de costos operativos, mejoras en la experiencia del cliente y un aumento en las oportunidades de generación de ingresos. Lo cual es coherente con los resultados y hallazgos de esta investigación.

\section{DISCUSION FINAL}

Como trabajo significativo de esta investigación se aporta a la comunidad empresarial y académica un método estructurado, el cual articula el concepto de calidad del servicio para evaluar integralmente la calidad del servicio documental en un centro de educación superior apoyado en Seis Sigma, de igual manera se pudo evidenciar como resultado del análisis de las métricas que la dimensión de calidad que obtuvo mejor desempeño fue 'Documentación de forma completa', lo cual se evidencia empíricamente con el nivel sigma que alcanzó el nivel más alto. Así mismo, la característica crítica del servicio fue la dimensión de calidad 'Respuesta pertinente' toda vez que disminuyo su desempeño de enero a febrero, presentando el nivel sigma más bajo. Sobre lo cual hay que implementar planes de mejora y seguimiento.

Así mismo, se puedo concluir que la mayoría de las dimensiones de calidad inherentes al servicio documental prestado estuvieron por encima del nivel esperado y que su rendimiento fue bueno, ya que mejoran de un periodo a otro. Sin embargo, se recomienda con base en los resultados de la evaluación de los dos periodos evaluar las buenas prácticas establecidas que incidieron en la mejora de desempeño de las métricas y replicarlas para los periodos futuros, toda vez que lo anterior permite consolidan la prestación del servicio en el centro de atención documental de la institución de educación. De igual manera los resultados de esta investigación muestran la pertinencia que existe para combinar y estructurar conceptos de calidad del servicio con las métricas de Seis Sigma, permitiendo diseñar un estándar del rendimiento del servicio para el 
mejoramiento de la calidad del servicio por medio de la contextualización de Seis Sigma. Lo anterior es replicable en cualquier organización de servicios a nivel nacional e internacionalmente.

\section{CONCLUSIONES}

De acuerdo al trabajo presentado y a los resultados obtenidos, se pueden plantear las siguientes conclusiones principales:

1.-La prestación del servicio del centro de atención documental presenta un buen desempeño, considerando que supera los criterios establecidos de rendimiento y nivel sigma para los dos periodos $(95 \%$ y 3$)$. Solo la estandarización de procesos alcanza un 89,27 \% en enero, lo cual se mejora significativamente en el segundo periodo con un rendimiento $\mathrm{Y}$ del $97,27 \%$

2.-Se aporta a la comunidad científica y a las empresas de servicios, un método estructurado que integra dimensiones de calidad de un servicio y la valoración de métricas de seis sigma a estas dimensiones, que permiten evaluar de forma puntual e integral el desempeño de un servicio.

3.-Pero lo mas relevante de ésta investigation, es que aporta un método, que asocia dimensiones de calidad y los criterios de valoración de estas dimensiones por medio de métricas de seis sigma. Lo cual es reproducible y applicable en cualquier servicio a nivel internacional

\section{REFERENCIAS}

Abdullah, F., Measuring Service Quality in Higher Education: HEdPERF versus SERVPERF. https://doi.org/10.1108/02634500610641543, Marketing Intelligence \& Planning, 24(1), 31-47 (2006)

Anthony, S. y Antony, J., Lean Six Sigma in Academic Institutions-UK vs. Rest of the World, Fourth International Conference on Lean Six Sigma for Higher Education, 68-82 (2017)

Antony, J., Lu, J. y Laux, C., Lean Six Sigma for higher education. https://doi.org/10.1108/IJPPM-09-2016-0195, International Journal of Productivity and Performance Management, 66(5), (2017)

Boon, T., Shukur, M., Ahmad, F. y Ahmad, N., Evaluation of Service Quality of Private Higher Education Using Service Improvement Matrix. https://doi.org/10.1016/j.sbspro.2016.05.417, Procedia - Social and Behavioral Sciences, 224, 132$140(2016)$

Bo, X., Wang, Y. y Zou, J., Implementation of Six Sigma to Service Quality Management in Auto After-Sale, The 19th International Conference on Industrial Engineering and Engineering Management, 1163-1171 (2013).

Chen, H.C., Chen , K.S. y Chang, T.L., An application of Six Sigma Methodology to Enhance Leisure Service Quality, Quality \& Quantity, 44(6), 1151-1164 (2010).

Chen, H., Wang, L., Mu, Y. y Wang, J., An Application Study of Lean Six Sigma in Logistic Service Quality Management, International Asia Conference on Industrial Engineering and Management Innovation (IEMI2012) Proceedings, 843-850 (2013).

Deshmukh, V.K., Mukti, S.K., y Agrawal, A., Applicability of Lean Six Sigma in Hospitals, Advances in Industrial and Production Engineering, Lecture Notes in Mechanical Engineering, 861-870 (2019).

Fontalvo, T.J., Aplicación de Seis Sigma en una empresa productora de Cemento, Escenarios, 9(1), 7-17 (2011).

Gadea-Cavazos, E., Morquecho-Sánchez, R., Pérez-García, J.A. y Morales-Sánchez, V., Adaptación del Cuestionario SERVQUAL para la Evaluación de la Calidad del Servicio Educativo en la Asignatura de Cultura Física y Salud en México, Cuadernos De Psicología Del Deporte, 18(3), 150-162 (2018).

Hsieh, YJ., Huang, LY. Y Wang, C.T., A Framework for the Selection of Six Sigma Projects in Services: Case Studies of Banking and Health Care Services in Taiwan, Service Business, 6(2), 243-264 (2012).

Hongoro, C., McPake, B. y Vickerman, P., Measuring the Quality of Hospital Tuberculosis Services: a prospective study in four Zimbabwe hospitals, International Journal for Quality in Health Care, 17(4), 287-292 (2005).

Johnstone, P.A., Hendrickson, J.A. y otros 5 autores, Ancillary services in the health care industry: ¿is Six Sigma reasonable?, Quality management in health care, 12 (1), 53-63 (2003).

Malik, M., Danish, R. y Usman, A. The impact of Service Quality on Students' satisfaction in Higher Education Institutes of Punjab, Journal of Management Research, 2(2), 1-11 (2010).

Miniati, R., Frosini F. y otros 6 autores, Experience of Lean Six Sigma Quality Approach to Hospital Laboratory Services, 6th European Conference of the International Federation for Medical and Biological Engineering, IFMBE Proceedings, 45, 609-612 (2015).

Nadeau, S., Lean, Six Sigma and Lean Six Sigma in higher education: A review of experiences around the world, American Journal of Industrial and Business Management, 7(5), 591-603 (2017). 
Otálora, M., Orejuela, A. R. y Hernandez-Espallardo, M., Propuesta Metodológica para medir la Calidad del Servicio de consulta externa en Medicina General, Estudios Gerenciales, 27(120), 185-204 (2011).

Rodríguez, F., Seis Sigma en una empresa de servicios de informática, Entre Ciencia e Ingeniería, 9(17), 56-61 (2015).

Rodríguez-Ponce, E., Pedraja-Rejas, L. y otros 3 autores, El impacto del sistema de Aseguramiento de la Calidad en el Servicio entregado por las Universidades Privadas en Chile, Ingeniare: Revista Chilena De Ingenieria, 19(3), 409-419 (2011).

Rodrigues, L.L., Barkur, G., Varambally, K.V.M. y Golrooy, M., Comparison of SERVQUAL and SERVPERF metrics: An empirical study. https://doi.org/10.1108/17542731111175248, The TQM Journal, 23, 629-643 (2011).

Shokri, A., Six Sigma in Supply Chain, Supply Chain Strategies, Issues and Models, 63-98 (2014).

Sporn, B., Building adaptive universities: Emerging organ.isational forms based on experiences of European and us universities, Tertiary Education and Management, 7(2), 121-134 (2001).

Surange, V., Implementation of Six Sigma to Reduce Cost of Quality: A Case Study of Automobile Sector, Journal of Failure Analysis and Prevention,15(2), 282-294 (2015).

Subbarayalu, A. V., Kuwaiti, A., Development of a six sigma rating scale for measuring the quality of work life of teaching staff working in saudi universities, International Journal for Quality Research, 11(2), (2017).

Torres, E., Escala propuesta para medir la calidad del servicio de los cines, Innovar, 20(36), 157-171 (2010).

Tumino, M.C. y Poitevin, E.R., Evaluación de la calidad de servicio universitario desde la percepción de estudiantes y docentes: caso de estudio, REICE. Revista Iberoamericana sobre Calidad, Eficacia y Cambio en Educación, 12(2), 63-84 (2014).

Yildiz, S.M. y Kara, A., Developing Alternative Measures for Service Quality in Higher Education: Empirical Evidence from the School of Physical Education and Sports Sciences, Proceedings of the 2009 Academy of Marketing Science (AMS) Annual Conference,185-185 (2015).

Yousapronpaiboon, K., SERVQUAL: Measuring Higher Education Service Quality in Thailand.

https://doi.org/10.1016/j.sbspro.2014.01.350, Procedia - Social and Behavioral Sciences, 116, 1088-1095 (2014). 
